\title{
Le patrimoine historique des télécommunications françaises : de l'« archive » matérielle à la profusion immatérielle
}

The historical heritage of French telecommunications: from the material "archive" to the immaterial profusion

\section{Michel Atten}

\section{(2) OpenEdition}

\section{Journals}

\section{Édition électronique}

URL : http://journals.openedition.org/artefact/655

DOI : 10.4000/artefact.655

ISSN : 2606-9245

Éditeur :

Association Artefact. Techniques histoire et sciences humaines, Presses universitaires du Midi

\section{Édition imprimée}

Date de publication : 15 juin 2017

Pagination : 95-110

ISBN : 978-2-7535-6525-8

ISSN : 2273-0753

Référence électronique

Michel Atten, « Le patrimoine historique des télécommunications françaises : de l'« archive » matérielle à la profusion immatérielle », Artefact [En ligne], 5 | 2016, mis en ligne le 15 novembre 2017, consulté le 05 mars 2020. URL : http://journals.openedition.org/artefact/655 ; DOI : https://doi.org/ $10.4000 /$ artefact. 655 


\section{Le patrimoine historique des télécommunications françaises : de l'« archive " matérielle à la profusion immatérielle}

Michel ATTEN ${ }^{*}$

\section{Résumé}

L'article propose de retracer la constitution, sur un temps long (deux siècles), d'une belle collection d'objets constitutifs des réseaux de télécommunications, contribution majeure au patrimoine national français de ce secteur. Elle est récente car, longtemps, l'idée ou le geste de conserver des objets techniques, des machines ayant permis de communiquer à distance n'a tenu qu'au hasard, à des individualités, voire à des lieux où l'on stocke les matériels obsolètes. Le seul dépôt notable auprès du Conservatoire des arts et métiers est lié à l'invention, non à la volonté de conserver la mémoire des moyens de communication.

Au début des années 1970, naît, à l'initiative d'ingénieurs, une démarche de collecte d'objets techniques utilisés dans les télécommunications. Marquant un besoin de mémoire, d'identité en des temps de profonde mutation de l'entreprise, elle s'inscrit également dans un mouvement large d'essor des préoccupations patrimoniales. La professionnalisation de l'équipe, depuis la fin des années 1990, permet l'enrichissement et le traitement des collections, mais elle ne convaincra pas les historiens de considérer les objets techniques comme une ressource, une « archive » permettant d'enrichir l'histoire.

Mots-clés : archive immatérielle, archive matérielle, collections, musée d'entreprise, patrimoine.

*. Michel Atten est directeur (h) des Archives et patrimoine historique du Groupe France Télécom/ Orange, historien, chercheur associé au laboratoire Techniques, Territoires et Sociétés (LATTS). Son domaine de recherche est l'histoire des sciences et des technosciences en société. Il a notamment publié «Ce que les bases de données font à la vie privée : l'émergence d'un problème public dans l'Amérique des années 1960 », Réseaux, n 178-179, 2013, p. 21-53, et « Les nouvelles formes de technoscience universitaire comme produits de la R \& D industrielle? L'exemple du transfert CNET-ENS Cachan ", Artefact, n 3 , 2015, p. 131-150. Contact : [michel.atten@u-pem.fr]. 


\section{Abstract. The historical heritage of French telecommunications : from the material "archive" to the immaterial profusion}

This article seeks to trace, over a period of two centuries, the building up of a large collection of objects of the French telecommunication networks. Today, this collection constitutes a major part of the French heritage in the domain of telecommunications. For a long time, the initiatives of conserving technical objects or machines for long-distance communication were sporadic, random, and based on the initiative of individuals or institutions holding obsolete equipments. As a result, a single collection of machines was donated to the Conservatoire national des arts et métiers in 1920 only to be incorporated in their collection in the framework of promoting the history of innovation or to incite students to be invention and innovation, rather than as repositories of a memory of telecommunication equipments.

At the beginning of the 1970s, engineers and workers of telecommunications - French PTT - started to collect telecommunication objects in the $R \mathcal{E} D$ center (CNET) in Paris. This initiative soon gave birth to a need for institutional memory and professional identity during periods of profound changes in the world of telecommunications. It further built on the rise of the interest in industrial heritage matters. As a consequence, starting in the late 1990s, a team (Records Management and Historical Heritage, now a service of Orange) has been getting more and more professional. This evolution makes it easier to expand and handle the collections but does not convince historians to look upon objects as resources or records that may enrich history.

Keywords : collections, digital records, heritage, material source, museum of business.

L'engouement pour le patrimoine est massif, comme en témoigne, en France comme dans beaucoup de pays, la multiplication des musées, espaces d'exposition, journées portes ouvertes organisées par les administrations, les grandes institutions des États ou les entreprises, les centres de recherche, les universités, etc. Pourtant, il convient, nous semble-t-il, de regarder les choses d'un peu plus près, de différencier l'emballement pour les ors des palais de la République de l'intérêt porté aux collections et aux musées techniques, par exemple, à la constitution et à la préservation de cette partie du patri- moine, ces choses qui tiennent une place croissante dans nos vies (pensons aux téléphones portables, aux smartphones, aux ordinateurs aujourd'hui) dans un monde où les entreprises privées deviennent prépondérantes. Doit-on suivre Patrick Fridenson quand il assure que, " pour beaucoup d'entreprises, qu'elles soient opérationnelles, qu'elles aient été absorbées ou aient disparu, le patrimoine et la mémoire sont devenus des enjeux massifs" ${ }^{1}$ ? " Les musées d'entreprise peuvent-ils être autre chose que des mémoriaux à la gloire des entreprises et du progrès technologique ${ }^{2}$ ? ». 
Doit-on se satisfaire de leur survie en des temps difficiles parce que, pour les entreprises (multinationales), « laisser tomber ces institutions muséales, désormais bien ancrées au plan local et régional, pourrait être contre-productif en terme $\mathrm{d}^{\prime}$ image et de communication ${ }^{3} »$ ?

Si les études et constitutions de patrimoine industriel et de musées d'entreprises ont connu un essor important dans les trente dernières années, très peu concernent le domaine des télécom- munications. Nous nous proposons de réfléchir, à travers l'exemple de France Télécom-Orange et de notre propre expérience ${ }^{4}$, en nous appuyant sur les travaux existants ${ }^{5}$, sur cet intérêt pour les musées d'entreprises, pour la conservation-valorisation du patrimoine industriel comme valeur partagée. Qu'entend-on au juste par conserver et valoriser le patrimoine, la mémoire, et quel est le rôle des musées techniques?

\section{Des hasards qui produisent des collections}

Si on l'observe sur un temps un peu long, cet intérêt pour le patrimoine ne semble guère s'étendre au domaine technique des systèmes de communication à distance qui sont, il est vrai, d'émergence relativement récente (fin $\mathrm{du} \mathrm{XvIII}^{\mathrm{e}}$ siècle). La première " collection de machines à communiquer » identifiée en France concerne, dans le dernier tiers du XIX ${ }^{\mathrm{e}}$ siècle, les dispositifs de télégraphie électrique accumulés par l'Administration des lignes télégraphiques, que cette dernière soit sous tutelle du ministère de l'Intérieur ou intégrée après 1878 au sein du nouveau ministère des Postes et Télégraphes. La réunion de machines télégraphiques, visibles par le public, concerne avant tout l'invention : il s'agit de rendre accessibles aux ingénieurs, inventeurs, bricoleurs, constructeurs d'appareils scientifiques ou techniques, fabricants et industriels de la métallurgie (fils, câbles) les terminaux et équipements de télégraphie électrique, les outils, appareils de mesure, qu'ils soient utilisés quoti- diennement dans le réseau public ou au stade de prototypes brevetés ou encore protégés par des notes cachetées déposées à $l^{\prime}$ Académie des sciences ${ }^{6}$. C'est une époque d'inventeurs individuels, d'émergence de la nécessité nouvelle de déposer des brevets, en rupture avec la période précédente où une télégraphie appartenant à l'État qui s'en réserve l'utilisation n'a pas besoin de brevets, comme en témoignent le système Chappe et les premiers télégraphes électriques ${ }^{7}$. De plus, l'Administration des lignes télégraphiques possède ses propres ateliers de fabrication de matériels dans lesquels furent développés, améliorés, adaptés et fabriqués, dans les premières décennies de la télégraphie, des systèmes nouveaux, rapides comme le Hughes ou le Baudot. Cette collection de télégraphes, présente dans les locaux et/ou les laboratoires de l'École supérieure des P. et T. de la rue de Grenelle ${ }^{8}$, perd son intérêt éducatif au début $d u x^{e}$ siècle, avec le développement de la téléphonie qui nécessite bientôt une production 
plus massive, qui draine rapidement l'intérêt des inventeurs et transforme les manières $d$ 'innover, lesquelles se font plus collectives, au sein d'ateliers ou d'entreprises. Le brevet d'invention, devenu d'un usage beaucoup plus systématique, avec son texte, ses schémas et plans, rend publiques les informations concernant le nouveau dispositif, faisant perdre au " musée des télégraphes » bien de son intérêt. C'est pourquoi cette collection, devenue numériquement importante et d'intérêt essentiellement de mémoire, est confiée par les PTT, au début des années 1920, au Conservatoire national des arts et métiers à des fins de conservation ${ }^{9}$.

Ce transfert doit également être mis en perspective avec les mutations des systèmes de communication électrique à distance de l'entre-deux-guerres : à partir des années 1920, émergent de nouvelles manières de développer les innovations par la coopération toujours plus intime entre science et développement. Un nouveau type de structure industrielle, qu'on va appeler la Recherche \& Développement, associe chercheurs issus directement $\mathrm{du}$ monde universitaire et ingénieurs, inventeurs, techniciens, qu'ils travaillent dans des unités ou laboratoires de recherche, dans les services d'exploitation ou dans les usines de fabrication des équipements; le système Bell, aux États-Unis, en est le représentant archétypique. C'est une époque caractérisée également par la spécialisation croissante des savoirs et savoir-faire des ingénieurs et techniciens des PTT : transmission, commutation, lignes à grande distance, câbles sous-marins, télégraphie et téléphonie sans fil, autant de directions et de services séparés, développant ou acquérant chacun ses dispositifs, ses appareils de mesure et de maintenance, ses terminaux. Cette mutation technique fait éclater la culture commune que possédaient davantage les télégraphistes du $\mathrm{XIX}^{\mathrm{e}}$ siècle - ils déroulaient leur carrière de service en service. En effet, elle incite les ingénieurs et administrateurs des entreprises et/ou administrations de ce secteur à se tourner toujours plus vers les innovations qui se multiplient. Cela est vrai aussi pour les PTT français qui, quoique avec un certain décalage par rapport aux pays les plus équipés (ÉtatsUnis, Europe du Nord...), connaissent un renouveau certain dans les années 1920 et 1930 .

Devenu un objet technique de masse (les terminaux se comptent désormais en centaines de milliers, voire en millions), le poste téléphonique est standardisé, une nécessité technique qui s'accompagne d'une uniformisation formelle généralisée : ces fameux postes en bakélite noire (le PTT-24 en France). En quelques années, on est passé d'une profusion de téléphones dotés de formes, de designs, d'élégances " arts-déco » faites de mélange de matériaux, à une production uniformisée, taylorisée, plastifiée de postes téléphoniques "modernes ", fournis tant par les PTT européens que par la grande compagnie privée américaine AT\&T.

Un tel changement durable (seulement trois types de postes en cinquante ans, PTT-24, U-43 et S-63) suscite-t-il au sein de cette grande administration un intérêt à conserver les magnifiques postes anciens, témoins de cette profusion qui fait le bonheur des musées, conservatoires et collectionneurs d'aujourd'hui? Nous n'en avons pas trouvé 
trace, pas plus que d'un quelconque intérêt patrimonial dans l'entre-deuxguerres pour un domaine qui connaît un renouvellement de plus en plus rapide de ses dispositifs et outils techniques, si l'on excepte la création du Musée postal en 1935, voué, il est vrai, essentiellement à la collecte des timbres. Ajoutons le fait que, jusque dans les années 1970, beaucoup des équipements achetés par l'administration française des PTT pour équiper ses réseaux proviennent de compagnies étrangères ${ }^{10}$. Cela constitue un élément peu propice à aiguiser le sentiment de fierté nationale qui accompagne souvent les inventions.

\section{Pourquoi une Collection historique au début des années 1970 ?}

La situation change en 1972. À l'initiative de quelques " techniciens " et notamment de Michel Jacquet, ingénieur des télécommunications passionné de techniques, le Directeur général des télécommunications, Louis-Joseph Libois, crée un service intitulé "Collection historique de matériels de télécommunication ", chargé de collecter " les matériels anciens ou actuels qui caractérisent les différentes étapes du développement des techniques de télécommunication, ainsi que les documentations s'y rapportant ». Il insiste explicitement pour que les « services extérieurs » y contribuent, compte sur « la collaboration de tout le personnel » et précise que le matériel doit être " comptabilisé et géré comme faisant partie intégrante du patrimoine des Télécommunications ${ }^{11}$ ». Il le rattache à la recherche, au Centre national d'études des télécommunications (CNET) pour des raisons pratiques, une plus grande souplesse budgétaire notamment (à cette époque, même le directeur (DGT) de l'une des trois grandes directions du ministère des PTT n'avait pas carte blanche pour ce genre de création $^{12}$ ), mais également parce que ce centre de recherche est une institution qu'il connaît très bien - il y a travaillé de 1941 à 1971 et en fut le directeur de 1968 à 1971 - et certains des initiateurs du projet y travaillent également.

Les années 1970 voient un bouleversement et un renouvellement important des équipements - la DGT commence à introduire massivement de l'électronique dans les réseaux - et les équipements obsolètes se multiplient. La Collection se développe aussi en vidant des armoires $^{13}$, en accueillant des dons et en achetant des collections privées d'objets techniques anciens, notamment de très beaux appareils de télégraphie et de téléphonie du xIX ${ }^{e}$ siècle (figures 22 à 30 , cahier couleur). Très rapidement, cette collection, installée dans le Service des expositions du CNET, à Issy-lesMoulineaux, présente ces objets au sein d'expositions popularisant les plus récents développements-inventions du CNET (des lasers aux semi-conducteurs, de l'optoélectronique à la commutation électronique et numérique, des systèmes MIC à la numérisation du signal 
de parole...) dans de nombreuses manifestations nationales et internationales (Télécom mondiales (UIT), SICOB, Salon de la physique, etc. $\left.{ }^{14}\right)$.

Mais pourquoi constate-t-on une institutionnalisation à ce moment et sous cette forme? Cette démarche subite est effectivement originale à plusieurs titres. Elle se fait en dehors des structures existantes qui auraient pu constituer un lieu "naturel » pour une telle initiative, comme le musée de la Poste, installé au centre de Paris, boulevard de Vaugirard, reconstruit depuis peu (1967) et qui accueillait déjà des archives des PTT (collections de timbres, d'objets du travail des postiers) et des objets du système de télégraphie Chappe et électrique. Elle est antérieure au grand mouvement qui accompagne la loi sur les archives de 1979, qui se traduit par le recrutement de la DGT d'une archiviste, Catherine Bertho. L'émergence de la Collection historique est également antérieure aux mouvements intellectuels, associatifs et politiques repérés par Florence HachezLeroy, entre autres, comme constitutifs du patrimoine industriel et qu'elle situe à la fin des années 1970 et au début des années 1980, accompagnant la vague de désindustrialisation postérieure à $1975^{15}$, ou même du grand mouvement intellectuel et collectif suscité par les « lieux de mémoire » chers à Pierre Nora.

Bien évidemment, cette initiative de 1972 s'inscrit dans un contexte technique bien particulier, celui de la mutation alors en cours de la téléphonie analogique vers le numérique; le premier autocommutateur téléphonique entièrement numérique vient d'être mis en service à Perros-Guirec en 1970, une première mondiale! Il s'agit d'une muta- tion extrêmement profonde et de grande ampleur puisqu'elle annonce en partie le monde des technologies de l'information et de la communication et du numérique d'aujourd'hui. Mais, comme on l'a vu pour l'entre-deux-guerres, une mutation importante, voire radicale, de l'appareil productif des télécommunications ne produit pas automatiquement des actions de conservation de la mémoire et de sauvegarde d'objets-archives.

La raison principale nous semble être la revendication identitaire des " télécommunicants » face aux postiers, une identité qui n'a rien de naturelle comme le croient Christian Chaunavel et Denis Varloot, mais dont la construction est à un tournant au début des années 1970. Cette démarche identitaire, sous forme d'autonomie des télécommunications par rapport à la Poste, n'est pas tout à fait nouvelle. Sans remonter plus avant, elle est cultivée par l'Association des ingénieurs des télécommunications (AIT), organe corporatiste représentant les ingénieurs du corps $^{16}$ depuis avant la Seconde Guerre mondiale ${ }^{17}$. Les années 1960 qui voient s'accentuer la crise $d u$ téléphone ${ }^{18}$, réaniment les revendications des ingénieurs qui multiplient délégations et audiences auprès du ministre des PTT, croisant le fer avec les administrateurs ${ }^{19}$. Ils commencent à oser des interventions publiques (plaquettes, livres, articles dans les journaux, en France et à travers la création d'une organisation internationale, la FITCE ${ }^{20}$ ), en élargissant toujours plus ce qui sépare les Télécom du reste des PTT, réussissant même à entraîner sur leur position d'autonomie une des organisations syndicales, la Fédération nationale des télécommunications (FNT), alors que tous 
les autres syndicats feront de l'identité service public-unité des PTT leur revendication centrale.

En 1972, le directeur général Libois continue les actions d'autonomisation de son prédécesseur Pierre Marzin : séparation des budgets et création d'une société de financement du téléphone spécifique Finextel (1970), suppression du Secrétariat général commun, séparation des parcs d'ordinateurs, des services du personnel, des directions d'enseignement supérieur (DESA et DEST), création d'une revue spécifique - la « Revue T » ou Revue française des Télécommunications - indépendante de la revue du ministère, Postes et Télécommunications (1971), création de nouvelles sociétés de financement du téléphone (Agritel, Creditel), de la collection historique des télécommunications (1972). Un projet de loi prévoyant la création de deux établissements publics (industriel et commercial), chacun avec son budget, sa programmation pluriannuelle, etc., est présenté au président Georges Pompidou et au gouvernement par le ministre des PTT, Hubert Germain, à la fin de 1973, mais le processus sera interrompu par la mort de G. Pompidou au printemps $1974^{21}$. Enfin, ultime confirmation, des articles « techniques » historiques présentent certaines des pièces de la nouvelle Collection dans chaque numéro de la nouvelle Revue $\mathrm{T}^{22}$. Cette revendication identitaire va très rapidement s'imposer avec l'arrivée, fin 1974, du nouveau Directeur général, Gérard Théry, proche $\mathrm{du}$ président Giscard d'Estaing, qui obtient les moyens financiers et politiques de réaliser la modernisation accélérée du téléphone (le fameux $\Delta \mathrm{LP}$ ). La Collection historique voyant dès lors sa légitimité culturelle fortement relativisée - l'identité télécommunication n'est plus à construire, elle est éclatante - perdure, mais elle ne résistera pas à une exigence, classique dans la vie des entreprises, de mètres carrés disponibles $^{23}$. À la fin des années 1970, le nouveau directeur du CNET, Maurice Bernard, la poussera dans des locaux déserts du grand bâtiment souterrain de Murat (Porte d'Auteuil). Quant à l'idée d'un musée des télécommunications, installé dans le trou des Halles en pleine transformation, elle s'envole ${ }^{24}$. En revanche, sensible au patrimoine « traditionnel ", Gérard Théry, devenu le directeur d'une institution puissante, premier investisseur de France, renoue avec la tradition des PTT du début du $\mathrm{xx}^{\mathrm{e}}$ siècle, prenant soin de lancer des concours d'architectes pour certains bâtiments de directions nouvelles ${ }^{25}$.

\section{Vers la création du musée de Pleumeur-Bodou}

Les années 1980 marquent une inflexion assez claire vers l'histoire, largement autant sinon davantage que vers le patrimoine industriel, tout en élargissant nettement l'éventail des approches et des points de vue. D'abord, avec l'arrivée d'un ministre des PTT de François Mitterrand, Louis Mexandeau (1981-1986), historien de profession, qui annonce un Comité pour l'histoire 
de la Poste et des Télécommunications en novembre 1982. Ce comité devient effectif en février $1983^{26}$, avec la participation notamment de Catherine Bertho, conservateur des Archives nationales chargée de mission à la DGT, et de Patrice Carré, plume de Jacques Dondoux, nouveau directeur général des télécommunications. Ils viennent de publier, avec Claudine Guerrier, un ouvrage remarqué, Télégraphes et téléphones, de Valmy au microprocesseur ${ }^{27}$. Sans être exhaustif, citons, parmi les activités de ce comité ministériel, un colloque co-organisé, en 1984, avec l'Institut d'histoire du temps présent sur l'action et le rôle des agents des PTT dans la clandestinité au cours du second conflit mondial ${ }^{28}$, une Histoire des télécommunications en France, publiée en 1984, et une exposition célébrant les Cent ans de téléphone public, en $1989^{29}$. Est également significatif le travail organisé entre 1983 et 1985 par le Centre de recherche : une première mouture d'une " histoire " technique des travaux du CNET depuis sa création en 1944, rédigée en 1974 par deux ingénieurs, Jacques Dondoux et R. Giblin, ne sera pas publiée ${ }^{30}$. Le projet est repris en 1983-1985, en recourant cette fois à un groupe de travail constitué d'historiens professionnels extérieurs appartenant à des institutions réputées (Francois Caron, de Paris 4, Antoine Prost, de Paris 1, le directeur Alain Lancelot et des chercheurs - Thierry Vedel, Jean-Yves Capul - du CEVIPOF (Science Po), des universitaires (Alain Bertho, Pascal Griset), MarieChristine Kessler du CNRS), placés sous la direction de François du Castel, directeur adjoint, et de Françoise Lavallard, directrice des Relations extérieures ${ }^{31}$.
Citons encore le colloque de 1987, «L'État et les télécommunications en France et à l'étranger, 1837-1987 », organisé avec le monde universitaire - École pratique des hautes études et université RenéDescartes -, mêlant histoire et réflexions contemporaines autour de la dérégulation en cours $^{32}$. L'ancien directeur général Libois, qui a rejoint la Cour des comptes en 1977, poursuit sa vision technique en publiant une Genèse et croissance des télécommunications fort bien documentée et affirmant clairement l'histoire d'une crise durable et permanente (la " guerre de cent ans ») du téléphone ${ }^{33}$.

Mais le geste inaugural des collections n'est pas oublié... Il a même été entendu en divers lieux de province (Nancy, Lille, Lyon, Bordeaux, Alsace...) où des associations de personnels et de retraités des deux directions, DGP et DGT, recueillent de nombreux objets obsolètes des services. Huit d'entre elles se regroupent à l'échelon national en février 1984, ce qui permet d'obtenir des subsides des Affaires sociales du ministère. Mais, dans le contexte de valorisation de l'histoire, elle prend le nom de FNARH (Fédération nationale des associations de personnel des PTT pour la recherche historique) et tourne ses travaux historiques surtout vers la télégraphie sémaphore de Chappe. Par ailleurs, l'idée de musée semble faire son chemin à la DGT, dans un contexte où l'idée de patrimoine industriel se développe. Portée entre autres par une association, le CILAC, créée en 1979, et par un large mouvement qui voit la prise en compte très progressive du patrimoine au sein du ministère de la Culture dans les années 1980, la création de $\mathrm{l}^{\prime}$ Institut national du patrimoine (INP $\left.{ }^{34}\right)$, 
quoique ce soit plutôt du côté des arts, des monuments historiques, des traditions rurales, voire bientôt des anciennes usines ou emprises industrielles ${ }^{35}$ que la prise de conscience du patrimoine se tourne. L'idée de musée est reprise par le Directeur général Jacques Dondoux, puis par son successeur Marcel Roulet.

Le premier est saisi d'un projet porté par des ingénieurs et techniciens du CNET Lannion, autour du Radôme de Pleumeur-Bodou (Côtes d'Armor), première station terrienne française ${ }^{36}$. C'est en effet en 1962 qu'est installée cette gigantesque antenne, achetée par le CNET à l'entreprise états-unienne qui construit celle conçue par les laboratoires Bell et installée à Andover (États-Unis). Or, ces antennes sont obsolètes depuis la fin des années 1960 et, en 1985, la démolition de la station-sœur
d'Andover commence. D'où l'idée de "sauver " la station française, symbole très fort au sein du CNET Lannion, en lui adjoignant un musée, un bâtiment dont les plans initiaux semblent imposants. Ils sont quelque peu réduits par le Directeur général Jacques Dondoux pour des raisons budgétaires ${ }^{37}$, mais aussi parce que l'idée d'un musée dans la région parisienne n'est pas abandonnée, ce qui serait cohérent avec l'assignation du futur musée de Pleumeur aux seules télécommunications internationales ${ }^{38}$. Il est surtout porté avec constance jusqu'à son ouverture en 1991 par le nouveau directeur, Marcel Roulet ${ }^{39}$, et par des élus et politiques bretons, notamment ceux de la région de Lannion, qui s'accordent toujours dès qu'il s'agit de soutenir un projet de développement local ou régional ${ }^{40}$.

\section{Les années 1990 : irruption de la « communication"}

Les années 1990 voient, à France Télécom comme dans beaucoup d'entreprises françaises, une montée en puissance de la direction de la communication $^{41}$. Cette direction va progressivement aspirer le service des archives, la collection historique, puis le musée de Pleumeur-Bodou et, enfin, contribuer à exprimer la voix de l'entreprise, son logo et le discours historique et/ ou de mémoire ${ }^{42}$. Les recherches historiques se font sur de nouveaux objets (les communications internationales ${ }^{43}$ ). Celles qui portent directement sur l'histoire de France Télécom sont plus rares : une journée d'études sur l'histoire de la recherche en télécommunications dans la France d'après-guerre, en 1995, qui essaie de développer le dialogue entre mémoire de témoins (des chercheurs de premier plan de l'époque) et histoire (des historiens des sciences et des techniques) et dont les actes sont publiés par la revue Réseaux ${ }^{44}$. Les associations de la FNARH organisent plusieurs journées d'études-colloques, publient un livre de référence ${ }^{45}$ et, dans ce cadre, montrent une vraie préoccupation pour le patrimoine de type « industriel » en restaurant plusieurs tours Chappe, notamment 
à Saverne (Bas-Rhin), Marcy-sur-Anse (Rhône), Sainte-Foy-lès-Lyon (Rhône), Jonquières (Aude). Enfin, c'est du côté des sociologues du CNET, travaillant notamment sur les usages des télécommunications et publiant une revue scientifique, Réseaux, dont l'audience croît régulièrement, que l'on trouve, dans les années 1990, des travaux d'historiens ${ }^{46}$. Rien de comparable toutefois avec le geste du nouveau directeur de la Poste, André Darrigrand, qui, en 1995, crée un Comité d'histoire de la Poste, appuyé sur des historiens universitaires et doté de façon conséquente pour permettre de financer des recherches visant à enrichir la compréhension de l'organisation au moment de sa mutation, elle aussi, vers une entreprise privée.

Comme souvent, ce qui provoque des bifurcations tient à des choses très terre-à-terre, ici un déménagement ${ }^{47}$. L'énorme bâtiment souterrain de Murat, porte d'Auteuil, intéresse subitement la branche Itinéris du téléphone portable en pleine expansion, d'où la nécessité de déménager en quelques mois, en 1999, la Collection historique et ses milliers de pièces. Un chantier difficile, d'autant plus que si un bon millier de pièces étaient identifiées, traitées, inventoriées, plusieurs milliers d'autres, couvertes de poussière, emplissaient des étages entiers, déposées là au fil des dons effectués depuis une vingtaine d'années. Ce transfert fait dans l'urgence oblige à un tri radical et à une réinstallation dans les locaux restaurés d'un ancien central téléphonique démonté peu avant, à Soisysous-Montmorency (Val-d'Oise). Étant en charge de ce chantier, nous nous inspirons des nouvelles réserves du Musée des arts et métiers pour organiser, avec des aides spécialisées, le nettoyage, l'inventaire et le classement des objets regroupés par salle - commutation, transmission, satellites et câbles sousmarins, cabines et appareils de mesure. C'est aussi l'occasion de procéder à une expertise scientifique de toute une série de pièces des $\mathrm{XVIII}^{\mathrm{e}}$ et $\mathrm{XIX}^{\mathrm{e}}$ siècles avec l'aide d'un spécialiste, Paolo Brenni ${ }^{48}$, et de passer systématiquement les pièces au détecteur Geiger.

\section{La constitution du pôle Archives et patrimoine historique (APH)}

Ce premier changement radical est bientôt suivi d'un second, en 2000, un appel à candidature interne pour diriger le nouveau service d'archives. En effet, en 1999, le secrétaire général, mécontent du fait que l'archivage vivant soit laissé aux divers services munis de la seule charte d'archivage, décide de créer un nouveau service d'archivage pour lequel il fait recruter une équipe et rénover les locaux d'une ancienne usine à Ivry-surSeine $^{49}$. La responsable de cette nouvelle activité étant partie en retraite, l'occasion est saisie de réunir dans une même entité archivage vivant, archivage historique, documentation et collection (qui dans notre esprit est une forme d'archivage, celui des équipements obsolètes dignes 
d'être conservés). Cette approche se rattache à nos réflexions et à nos travaux antérieurs et aux travaux d'histoire des sciences mettant en évidence l'importance de l'instrumentation, de la mesure, des pratiques et des gestes quotidiens et des activités de $\mathrm{R} \& \mathrm{D}$ dans le développement des sciences et des techniques ${ }^{50}$. Notre proposition une fois actée ${ }^{51}$, le premier mouvement sera d'envoyer en urgence presque tous les personnels issus des divers services antérieurs suivre des formations spécialisées, au CNAM notamment. Le nouveau service baptisé désormais "Archives et patrimoine historique » organise autant que possible des passerelles entre activités : par exemple, la récupération d'équipements de l'exploitation doit s'accompagner du recueil de la documentation, technique, commerciale, marketing, de la Dircom, sous forme papier et/ ou électronique et confiée à la section Documentation $^{52}$; ou, pour la section Vidéo, outre les appareils pour les lire, la collecte de films, vidéos et notamment d'une collection très riche mais dispersée de documents audiovisuels supports des VIF... Ces derniers, très utilisés par diverses directions de France Télécom lors du « débat » interne lancé par le rapport Prévôt de 1989, préparant le changement de statut de France Télécom à la fin des années 1980 et dans les années 1990, sont menacés par la fermeture de plusieurs services dans les années 2000. Côté extérieur, APH propose une collaboration (matérielle et intellectuelle) avec le musée de Pleumeur-Bodou, pérennisé par le fait que son antenne-Radôme est classée "Patrimoine du $x^{\mathrm{e}}$ siècle » en 2004; entraide avec les associations et « musées » FNARH (conseils, échanges de pièces contractualisés, transmission d'outils informatiques pour inciter ces associations à réaliser de vrais inventaires), puis bientôt récupération de collections menacées par les fermetures de sites. Enfin, démarches auprès de certaines collectivités locales ou régionales en vue de créer un musée des télécommunications dans la région parisienne.

En bref, APH était organisé pour offrir à tous les intéressés et particulièrement aux historiens, des outils, du matériel, de la documentation, des archives, des ressources matérielles, imprimées, photographiques, vidéographiques, etc., permettant d'étudier la construction d'une infrastructure de communication, la combinaison de divers réseaux et services plus ou moins interconnectés ou reliés dans leur déploiement géographique et social ${ }^{53}$. Car l'enjeu de la constitution d'un tel patrimoine, les travaux de David Edgerton ${ }^{54}$ le soulignent, est de conserver les objets-témoins phares des principales innovations du secteur (un audion, un laser, un faisceau hertzien, une fibre optique multimodale ou une antenne de satellite monumentale...), mais aussi ceux qui permettent d'étudier les techniques, ici celles des systèmes de communication à distance, dans l'histoire, dans leurs déploiements longs, matériels et sociaux indissociables. L'histoire des techniques en société n'est pas celle des innovations auxquelles souvent on les cantonne, ce qui pourrait permettre de comprendre, par exemple, pourquoi ce qui nous est apparu comme une évidence crevant les yeux dans les années 1960-1970, le retard du téléphone français, n'a pas été perçu de la même façon par les générations antérieures. 
L'intégration des archives vivantes au sein d'APH eut un autre effet spectaculaire : la prise de conscience, en 2002, que la quasi-totalité de l'information circulant dans l'entreprise prenait désormais des voies numériques et que rien n'était prévu pour en conserver la mémoire ${ }^{55}$. Cela nous conduisit à monter un projet " Archivage et gestion de l'information " $(\mathrm{AGI})^{56}$, validé et lancé en avril 2005 par le Comité exécutif d'Orange, avec un comité de projet regroupant des représentants de toutes les grandes divisions d'Orange-France sous la direction du secrétaire général. La mise en place d'une politique d'archivage numérique globale pousse à ouvrir une réflexion sur la conservation des produits nouveaux, d'ores et déjà en place, voire pour certaines parties obsolètes, les services dits «immatériels ».

Il n'est pas sûr que les résultats atteints en $2007^{57}$ aient été à la mesure des efforts déployés. Le collationnement a plutôt bien marché, avec la complicité de nombreux collègues d'Orange, avec les associations d'anciens, ce qui traduit un attachement persistant aux dispositifs, terminaux, équipements, etc. qu'ils ont maniés et entretenus durant leur carrière. Mais, du côté de la recherche historique et patrimoniale, l'engouement des universitaires ne fut pas du même $\mathrm{cru}^{58}$. Les travaux sur les notions d'infrastructure, voire d'infrastructure numérique de connaissance sont d'actualité (sans elle, il n'y aurait pas de science du climat, par exemple ${ }^{59}$ ), et une attention croissante est portée à l'indispensable prise en compte des outils, dispositifs et procédures matériels pour rendre compte de l'innovation des technosciences, des techniques « immatérielles » ou du gou- vernement des hommes et des choses (notamment avec les travaux des Science Studies ou des STS). Mais encore très rares sont les historiens, les sociologues, les universitaires des sciences sociales, etc., venus les voir, décidés à s'y intéresser, à se servir de ce type d' " archives " beaucoup plus larges et riches que les seules concernant les innovations. Nous n'avons pas trouvé trace de débats, de réflexions, au sein de l'entreprise ou dans sa proximité historienne, sur la spécificité du patrimoine historique, comme $s^{\prime}$ il était un simple prolongement du patrimoine vivant comme le définit la note de Libois de 1972, sans dire ce qu'il est : le réseau, l'infrastructure-monde, le service, les usages, les membres du personnel, tout cela à la fois ${ }^{60}$. De plus, les réductions drastiques, en personnel et en moyens, instaurées par la direction du Groupe depuis quelques années (en particulier durant la sombre époque Breton-Lombard, héritiers, il est vrai, d'une gestion hasardeuse) ont largement entravé depuis les développements réalisés au début des années $2000^{61}$.

Je voudrais terminer cette mise en perspective par une remarque concernant la notion de patrimoine matériel à travers deux " objets-dispositifs techniques » récents, choisis à titre d'exemples, dont la conservation future me semble poser des questions nouvelles. L'actualité de la notion d'infrastructure, objet d'attention des sciences sociales depuis quelques années, semble particulièrement difficile à saisir en termes de patrimoine, tant par ses dimensions géographiques (le réseau planétaire?) que par la part croissante du soft, de cette forme particulière du matériel qui pose de nouveaux défis aux conservateurs de patrimoine : que 
signifie conserver une infrastructure? Un satellite ou une station terrienne donne l'idée d'un réseau satellitaire, même si la question se posait déjà dès cette époque : souvenons-nous que, dès le début, la première offre de service commercial de communication par satellite en 1964 fut celle d'un réseau mondial (Intelsat). Mais conserver l'infrastructure et la mémoire du net, du web, du « cloud computing $»^{62}$ ? La notion de patrimoine ne serait-elle plus liée automatiquement à un pays, à une nation?

L'autre « objet », encore plus répandu, est tout aussi étrange. Que faut-il dire? Téléphone portable, Smartphone, terminal nomade de communication capable depuis presque n'importe où, d'envoyer ou de réceptionner des SMS ou des MMS, de se parler, de prendre des photographies et des vidéos, d'écouter de la musique, de participer à des jeux multijoueurs en ligne, de lire son journal ou un manuscrit grec ancien dans l'autobus ou en avion, d'utiliser hors ligne les services, bref, ce petit « objet » reproduit en quelques milliards d'exemplaires (près de 7 milliards de portables en 2012)... On a besoin, pour en préserver un type, disons l'iPhone pour le plus connu, d'un beau petit écrin pour l'objet matériel et d'un système d'archivage électronique capable de préserver ad aeternam la simulation informatique d'un bon nombre des quelque 500 ou 600 applications, des simulations qui devront subir régulièrement des « migrations » permettant de prendre en compte les évolutions des logiciels et des supports de conservation. Préserver le patrimoine des services grand public offerts par des infrastructures technologiques passera bien par l'alliance des savoirs et des savoir-faire des conservateurs et des archivistes, mais aussi par ceux des spécialistes des systèmes d'information, des records managers, des historiens et des personnels des entreprises... Tout un chantier.

\section{Notes}

1. Patrick FrIDENSON, « Les entreprises comme potentiels d'action, au cœur des sciences sociales ", Tracés. Revue de sciences humaines, horssérie, 2010, [http:/ / traces.revues.org/4676].

2. Paul RAsse, "Les musées d'entreprise : quelle médiation de l'histoire? ", Communication et organisation, $\mathrm{n}^{\circ} 7,1995$, [http://communicationorganisation.revues.org/1768].

3. Pascal Griset, Léonard LAborie, « D'entreprise ou de société? Deux opérateurs "historiques" et leurs musées, EDF et Orange ", Hermès, $\mathrm{n}^{\circ}$ 61, 2011, p. 119-124, [http ://www. cairn.info/revue-hermes-la-revue-2011-3page-119.htm].

4. Reprenant la communication faite en 2011 au colloque "Cabinets de curiosités, collections techniques et musées d'arts et métiers : origines, mutations et usages, des Lumières à la Seconde Guerre mondiale ", ce texte s'efforce, comme travail historique, de s'appuyer sur des textes, des décisions, des références, et aussi, compte tenu de la proximité temporelle des événements retracés, sur la mémoire (la nôtre ou celle d'autres anciens acteurs), en étant tout à fait attentif au fait que mémoire ne fait pas histoire.

5. Notamment Denis VARLOot, Christian Chaunavel, Pascal Griset, Léonard Laborie, "Mémoire, identité, patrimoine : l'exemple de France Télécom », Flux, n 58, 2004, p. 71-78.

6. Voir les photos des appareils de mesure, étalons, etc., sous verre dans Notices descriptives sur quelques installations récentes du service des PTT, Paris, Imprimerie nationale, 1910, p. 250 et suiv.

7. Un des premiers brevets d'invention déposés en France est celui de l'américain Samuel Morse; il est pendant longtemps le seul ou presque.

8. Pour l'histoire de l'école supérieure des PTT, François GuILLET, École nationale supérieure des PTT, 1888-1988 : histoire de la naissance et de la formation d'un corps de l'État, Paris, Hervas, 1988, et Michel Atten, François du CAstel, Marie Pierre (dir.), Les Télécoms. Histoire des écoles supérieures des télécommunications. 1840-1997, Paris, Hachette, 1999.

9. Cette collection est inventoriée en 1920 sous les numéros 14601 bis à 16221. Elle reste peu 
connue, hormis le livre de descriptions techniques publié par un ingénieur de l'Administration, Émile Pierre Guillaume Montoriol, inspecteur des Postes et Télégraphes, professeur à l'École des PTT, nommé conseiller technique au Conservatoire des arts et métiers. Montoriol est alors en charge de l'aménagement de la galerie de télégraphie et de téléphonie. Émile Montoriol, La technique télégraphique en France, depuis l'origine, Paris, Librairie de l'École spéciale des travaux publics, 1916, [http://cnum.cnam.fr/CGI/redir. cgi?M13543]; Émile Montoriol, Conservatoire national des arts et métiers, Musée, Galeries de télégraphie et de téléphonie, Coulommiers, impr. Paul Brodard, 1921, 32 p., [http:/ / cnum.cnam.fr/CGI/ redir.cgi?M12531].

10. International Telegraph \& Telephone, Siemens, Ericsson.

11. Circulaire $\mathrm{n}^{\circ} 11$ du 13 mars 1972, signée du DGT (copie fournie par P. Battiston que nous remercions). Le comité de direction comprend deux ingénieurs généraux, Rouault à l'Inspection générale et Blanchi au CNET, un ingénieur en chef au CNET, M. Jacquet, un administrateur HC à la DGT, Masson, et un directeur départemental, Chapel, chargé du Service du cinéma et des expositions, ce qui souligne le sérieux de l'affaire.

12. C'est en tout cas l'explication rapportée par C. Chaunavel et D. Varloot dans leur interview, confirmée par L.-J. Libois à l'auteur, D. VARLOOT et al., " Mémoire, identité, patrimoine : l'exemple de France Télécom », art. cit.

13. Dont certaines, notamment dans l'énorme centre de la rue des Archives (75003), recélaient beaucoup d'objets-outils des années 1890-1930, témoignant de gestes de préservation anciens mais isolés.

14. On en trouve trace notamment dans les « rapport d'activité » annuels du CNET (Archives Orange). Ainsi se noue très tôt une proximité entre collection d'objets anciens et innovations.

15. Florence HACHEZ-LERoY, «Quarante ans de patrimoine industriel en France. Introduction », Patrimoine industriel, $\mathrm{n}^{\circ}$ 66-67, 2015.

16. Au sens étroit du terme, ceux sortant de l'École polytechnique dans le corps Télécommunications; voir M. ATteN, « Les ingénieurs des télécommunications (1844-1999) : un grand corps? ", in Pascal Griset (dir.), Les ingénieurs des Télécommunications dans la France contemporaine. Réseaux, innovation et territoires ( $\mathrm{XIX} \mathrm{X}^{e} \mathrm{XX} \mathrm{X}^{e}$ siècles), Paris, Comité pour l'histoire économique et financière de la France, 2013, p. 17-32.

17. Durant la Seconde Guerre mondiale, profitant de l'affaiblissement des administrations traditionnelles, les ingénieurs contribuent à la division de l'École supérieure des PTT en deux (ENST et ENSPTT) en 1942, à l'affirmation dans les régions, du rôle des ingénieurs par rapport aux directeurs départementaux (postiers).

18. Rappelons que le réseau français ne comptait, en 1965, que 6,1 lignes d'abonnés/100 habitants, quand la Suède en comptait six fois plus, et que le réseau français était classé selon le classement téléphonique de "type Shanghai » au $17^{\mathrm{e}}$ rang européen.

19. Voir, par exemple, Cahiers d'études et d'informations, $\mathrm{n}^{\circ}$ 51, 1966, revue de $\mathrm{l}^{\prime}$ Association des élèves et anciens élèves de $1^{\prime} E N S P T T$, qui montre des administrateurs épousant pleinement le constat commun de crise aigüe du téléphone, mais quelque peu choqués de l'agressivité de leurs collègues des télécommunications.

20. Fédération des ingénieurs des télécommunications de la communauté européenne.

21. Marie CARPenter, La bataille des Télécoms. Vers une France numérique, Paris, Economica, 2011.

22. Entre le 16 novembre 1971 et le $4^{\text {e }}$ trimestre 1974, chaque numéro comporte un article « Le téléphone et sa chronique ", signé par les créateurs de la Collection, Michel Jacquet et Pierre Lajarrige, avec pour certains l'aide de Gaston Letellier du CNET Paris.

23. C'était déjà le cas en 1922 pour le «musée télégraphique ", chassé de la rue de Grenelle pour laisser place au nouveau Service d'étude et de recherche technique (SERT), créé par Étienne Clémentel en 1916. Et de nouveau très à la mode depuis quelques années (bureaux paysagers, télétravail, bureaux de passage, nouveaux espaces collaboratifs - plusieurs travaux de sociologues publiés ou en cours).

24. Nous croyons savoir qu'un dossier existe aux Archives nationales, dans le fonds G. Théry, sans avoir pu encore y accéder.

25. D. VARLOot et al., " Mémoire, identité, patrimoine : l'exemple de France Télécom », art. cit. Pour le début du $\mathrm{xx}^{\mathrm{e}}$ siècle, Notices descriptives sur quelques installations récentes..., op. cit., notamment les photographies, p. 267 (Dijon), 275 (Boulognesur-mer), 278 (Paris 96).

26. Claude Perardel (dir.), Postes et Télécommunications françaises. Une chronologie du $\mathrm{Xx}^{e}$ siècle, Paris, FNARH, 2003, p. 402 et 405. Le caractère quelque peu artisanal de cet ouvrage rend la remontée aux sources premières complexe.

27. Paris, Le Livre de poche, 1981 ; cet ouvrage est quelque peu teinté de la culture " maison DGT » dominée par la mémoire et les points de vue des ingénieurs des télécommunications.

28. L'CEil et l'oreille de la Résistance : action et rôle des agents des PTT dans la clandestinité au cours du 
second conflit mondial. Actes du colloque tenu à Paris les 21-22-23 novembre 1984, Toulouse, Erès, 1986.

29. Le Téléphone au fil du temps : 1889-1989, cent ans de téléphone public, exposition du 12 décembre 1989 au 4 janvier 1990, Musée d'art moderne de la Ville de Paris, accompagnée d'un livre publié par Catherine Bertho et France Télécom (Direction générale).

30. Respectivement directeur du CNET et ingénieur général au Comité consultatif des Télécommunications de l'Union.

31. Ce travail, interrompu par l'éviction de Jacques Dondoux de la DGT et de François du Castel du CNET par la nouvelle majorité issue des élections de 1986, sera finalement publié avec des compléments en 1990 : Philippe Bata et JeanPaul Bloch, Le CNET. 1944-1974. Genèse et croissance d'un centre public de recherche, Paris, CRCT, 1990.

32. L'État et les télécommunications en France et à l'étranger, 1837-1987, Genève, Droz, 1991.

33. Louis-Joseph Liвois, Genèse et croissance des télécommunications, Paris, Masson, 1983. Pour une critique de cette vision d'une " crise centenaire ", nous renvoyons à Michel AtTEN, « Les ingénieurs des télécommunications entre science et gouvernance (1840-1940) », à paraître en 2017.

34. Entre autres, Jean-Michel LÉNIAUd, "Conclusion générale ", in Les métiers du patrimoine en Europe. Évolutions, enjeux, professions, colloque international des 21 et 22 janvier 2005.

35. Voir, à cet égard, la politique de Margaret Thatcher des années 1980 cherchant à instrumentaliser le patrimoine comme compensation aux casses industrielles massives produites par sa politique libérale abrupte, Stana NenAdic, " Historiens et patrimoine en Grande-Bretagne », Le Mouvement social, $\mathrm{n}^{\circ}$ 200, 2002, p. 116-122.

36. Pour ce paragraphe, nous nous appuyons essentiellement sur le récit Varloot-Chaunavel, sans pouvoir être toujours plus précis. Notons que, curieusement, cet article n'emploie jamais le terme de "patrimoine industriel ", ni ne cite le CILAC que Denis Varloot connaît pourtant très bien puisqu'il en est le président d'honneur en 2008. Voir [http://iuhps.org/conferences/ conf2008/20080313cpstcscnam/programme.pdf], consulté le 3 décembre 2016.

37. Il semble que le projet initial était inspiré par un projet encore plus grandiose, celui de La Villette.

38. D. VARLOOT et al., « Mémoire, identité, patrimoine : l'exemple de France Télécom », art. cit. Leurs souvenirs attribuent la décision d'un musée au ministre Louis Mexandeau en 1982, mais est-ce déjà le breton? En l'absence d'archives dispo- nibles actuellement, nous laissons la question en suspens. En revanche, le Centre de télécommunications spatiales a été transféré logiquement du CNET Lannion à la Direction des télécommunications internationales (DTRI), dès la fin des années 1960, et la référence de Chaunavel, à la fin des années 1980, au directeur des services radioélectriques, Lhermitte, en retraite depuis 1964, semble anachronique.

39. D. VARLOot et al., «Mémoire, identité, patrimoine : l'exemple de France Télécom », art. cit, p. 73.

40. Majorité et opposition, quelles que soient les alternances.

41. Voir, par exemple, R. Laufer qui constaste « la multiplication de directions de la communication rattachées le plus souvent à la direction générale des grands groupes", dans "L'entreprise : communication et légitimité », Réseaux, vol. 9, $\mathrm{n}^{\circ}$ 50, 1991.

42. Voir, par exemple, Patrice CARré et MarieDominique Leclère, France Télécom, mémoires pour l'action, Paris, France Télécom-Direction de la Communication, 1993.

43. Thèse de Pascal GRISET, Entreprise, technologie et souveraineté : les télécommunications transatlantiques de la France (1869-1954), université Paris 4, 1993.

44. Michel Atтen (dir.), Histoire, recherche, télécommunications. Des recherches au CNET. 19401965, [http://www.persee.fr/issue/reso_09845372_1996_hos_14_1].

45. Collectif FNARH, La télégraphie Chappe, Éditions de l'Est, 1993.

46. Notamment le $\mathrm{n}^{\circ} 49$, « Histoire des télécoms » (1991) ou le n 55 avec trois articles d'histoire sociale (1992).

47. Cette dernière partie, très récente, s'appuie essentiellement sur notre mémoire.

48. Expert international; voir, par exemple, l'Association de sauvegarde et d'études des instruments scientifiques et techniques de l'enseignement, [http://www.aseiste.org/invalide/assuite. php? menu $=2$ \& numero $=2]$.

49. Ce qui témoigne que l'on raisonne encore en terme de papier, ce qui est normal compte tenu que la totalité des documents contractuels nécessitent des signatures authentiques qu'on ne sait faire alors que sous forme papier.

50. Comme chercheur associé au Centre de recherche en histoire des sciences et des techniques (CRHST) de la Villette dirigé alors par Dominique Pestre. Voir, par exemple, Dominique Pestre, Introduction aux "Sciences Studies », Paris, La Découverte, 2006, chap. 4. 
51. Avec l'aide d'un manageur très sensible à l'intérêt de conserver ces collections, Patrick Roisin.

52. La section documentation récupère de magnifiques collections d'affiches, des milliers de cartes téléphoniques couvertes de publicité dignes d'un annuaire professionnel, mais elle ne parviendra pas à collecter la mémoire des campagnes publicitaires.

53. Avec l'ubiquité permise par les communications actuelles, espace de présentation et collection peuvent aisément coopérer, même séparés par des centaines de kilomètres.

54. David EDgerton, The shock of the Old. Technology and global history since 1900, Londres, Profile Books, 2006.

55. Là encore, de petits faits servent d'alarmes... Sollicités par la direction juridique de retrouver les auteurs d'un contrat, nous faisons un constat radical : notre incapacité de reconstituer l'organigramme de services modifiés moins de deux ans auparavant. L'organigramme, alors en ligne, est actualisé au fil de l'eau, ce qui est très efficient compte tenu du rythme des changements, mais qui écrase les versions antérieures, sans en garder la mémoire.

56. Avec, entre autres, l'aide inestimable d'une experte, Marie-Anne Chabin, par ailleurs chargée de cours au CNAM.

57. Année de notre départ.

58. Les objets, pas plus que la Nature, ne parlent pas tout seuls.

59. Pensons notamment aux travaux de Paul N. EDWARDs, A vast machine. Computer models, climate data, and the politics of global warming, Cambridge, The MIT Press, 2010.

60. Nous nous incluons dans ce constat.

61. Il n'a plus aujourd'hui (en 2016) qu'une seule personne bien isolée pour gérer la Collection.

62. Sans ignorer les recherches et actions entreprises, nous pensons ici aux services offerts par l'entreprise, à ses portails web par exemple, qui ont déjà totalement changé de nombreuses fois depuis 1996, sans la moindre conservation (du moins organisée). 